\title{
The impact of faulty prostatic phylogeny and the development of benign prostatic hyperplasia
}

\author{
Roper WG* \\ Private researcher, 21/33 Bernard Rd., Padstow Heights, Australia
}

\begin{abstract}
This is a complex disease which is influenced by so many different factors particularly including phylogenetic error affecting the transitional prostatic zone. This error renders this zone vulnerable to infection from the beginning of middle age. Its understanding commences with the deliberation that the first substance involved in the development of the disease is Phospholipase $\mathrm{D}$ which is attached to the inside of the outer membrane surrounding the germ Escherichia Coli by ionic and hydrophobic bonds. (Only some gram negative and no gram positive bacteria possess this outer membrane). Its main purpose there, is to control the curvature of this membrane. Lytic destruction of this germ results in the release of phospholipase $\mathrm{D}$. Here, its strong negative charge is neutralised by $\mathrm{Ca}^{2+}$ ions in the prostatic secretion and as such and being non-protein bound, it is able to undergo passive diffusion through the prostatic epithelial barrier into the substance of the transitional zone of the prostate. Here, it is converted to phosphatidic acid and lysophosphatidic acid. The latter acts as a vicarious growth factor which directly stimulates prostatic smooth muscle to undergo hyperplasia. It also stimulates the connective tissue of the transitional zone to produce a gene called Cyr61 which acts as a secreted autocrine and paracrine mediator in stromal and epithelial hyperplasia respectively. The latter is thought to be under feedback control through bi-directional channels.
\end{abstract}

There are two certain factors in the development of this disease, namely (a) The disease only affects the transitional lobe of the prostate and (b) The disease commences only after middle age begins, which condition dictates the start of the involution of the prostate gland resulting in the decline in the amount of the proven prostatic antibacterial factor zinc (see Text). These two factors must be kept constantly in focus in any discussion of this disease.

Their explanation per se, is enough to convince most people of the veracity of this manuscript. Further experimental proof leaves absolutely no doubt.

Finally, it must be remembered that testosterone/dihydrotestosterone must be present in adequate amount for the disease to exist and those with higher levels will usually have more severe disease. However, the degree of the disease is governed more by the number of receptors for Escherichia Coli which a male person possesses and this is at least in part, genetically determined. The degree is also governed by the number of times they sexually ejaculate after middle age commences.

\section{Introduction}

Sometime between 100 and 200,000 years ago, a phylogenetic error was made in the development of the human prostate gland. This error affected only the transitional zone of the prostate by creating direct right-angle entry of its ducts into the prostatic urethra as far as the verumontanum. At maturity, this makes it very easy for urine to enter the transitional zone. In addition, urothelial type epithelium (similar to the bladder) lines the prostatic urethra also as far as the verumontanum and extends into the ducts of the transitional zone for variable distances. Below the verumontanum, it changes to patchy pseudostratified or stratified columnar type which is more resistant to infection. The ducts of the peripheral zone enter the urethra at an angle due to the 35 degree forward bending of the urethra below the verumontanum. This angulation appears sufficient to prevent urine from entry into the ducts of the peripheral zone. The second factor is that the ducts of the transitional zone exhibit more branching and acinar proliferation than the other peri-urethral ducts. There is an unusual juxtaposition of glandular and stromal elements.

"Ontogeny recapitulates Phylogeny" is a well-known saying which means it is necessary to study prostatic embryology in order to understand the development of the prostate gland from its phylogenetic inception to maturity. The development of the embryonic stage is reflected by the interaction of 4 critical units: the Wolffian and Mullerian ducts,the urogenital sinus(UGS) and the fetal gonad, The prostate starts with the formation of prostatic buds from the fetal UGS into the urogenital mesenchyme (UGM) but the prostate does not really grow until puberty under the influence of androgens.

Nature enters the picture here and creates confusion by producing fusion of all human prostatic elements into a solid blog of tissue without identifiable lobes as in other species. McNeal could find no evidence of true anatomical lobes and instead described and defined 3 major glandular zones: central and peripheral (these two harbour most prostatitis and carcinoma respectively) and the transitional (which harbours benign prostatic hyperplasia BPH). This assessment has been accepted throughout the world. In this manuscript we are dealing only with the transitional zone (including the submucous zone) and $\mathrm{BPH}$.

Asymptomatic bacteriuria is rare in men before middle age but begins to happen from this time. There is also a sixfold increase in the number of white cells in prostatic secretions of men with BPH compared to healthy men. After involution of the prostate begins in middle age, $\mathrm{E}$. Coli germs are carried into the transitional zone via urine which readily enters it. This is proven by calculi which develop within it from middle

*Correspondence to: Roper WG, Private researcher, 21/33 Bernard Rd., Padstow Heights, Australia, Tel: 0415570 202; E-mail: w.roper@bigpond.com

Key words: phylogenesis, infection, growth factor, zinc

Received: September 20, 2019; Accepted: September 27, 2019; Published: September 30, 2019 
age. Some of the nuclei of these calculi contain infective material but there is no struvite present within it, indicating that E. Coli is the culprit. Some of the stones contain material which is made from urine alone and some are just amyloid. These stones do not appear in the other zones. Most of the entering E.Coli tend to colonise on the surface of the epithelium of the transitional zone. Prostatic/ejaculate fluid has also been shown by experiment to enter this zone during ejaculation [1] and because of its profound sterilising effect resulting in the release of Phospholipase D (PLD) which then diffuses into the transitional zone where it is converted to the growth factor Lysophosphatidic Acid (LPA).

The reason germs appear at this time is because middle age results in the beginning of involution of the prostate which causes reduction in the production of the prostatic antibacterial factor (P.A.F) zinc. Ion probe and mass spectrographic analysis have proven that zinc is the antibacterial factor. Exogenous zinc, given orally, does not increase the prostatic tissue zinc levels. Normally, these levels are 500-1000 times greater in the prostate substance than serum zinc levels. It is extremely important to note that in $\mathrm{BPH}$,there is a remarkable decrease in tissue zinc of $61 \%$ associated with a $20 \%$ increase in the urinary zinc/ creatinine ratio [2]. This defensive secretion of zinc antibacterial factor is even further evidence that infection by colonising E.Coli is taking place. This cycle of repeated colonisation and destruction results in the very slow growth which occurs in $\mathrm{BPH}$ and this is why there are no mitotic figures evident in the disease production.

In a Scandinavian experiment, LPA was shown to directly stimulate hyperplasia of cultured prostatic smooth muscle in a nutrient solution in direct proportion to the quantity of LPA supplied. It also decreased apoptosis. Prostatic smooth muscle is approximately $85 \%$ of the pathology of BPH [3].

At a later date, a combination of Japanese and American elite researchers showed that the remaining pathology is gleaned from the LPA stimulation of prostatic connective tissue, where it stimulated the production of a gene called CyR61 (Cysteine Rich and showing 6 as the last of the 6 genes in the CyR group) which functions in cell proliferation, differentiation and extracellular matrix synthesis. It acts as a mediator for the production of autocrine and paracrine hormones which caused stromal and epithelial hyperplasia respectively, which is evident in $\mathrm{BPH}$. This was confirmed by the much increased presence of G-protein receptors and by DNA microarray analysis of both stromal and epithelial cells [4]. CyR61 was found to be related to the development and progression of BPH regardless of symptoms.

In spite of the long periods of repeated exposure to LPA, there is not a semblance of immune processes developing towards it, which would affect its biological activity. This is because it is not a secreted toxic substance from E.Coli but rather an accidental repeated addition. BPH.

This then, completes the etiological process and development of

\section{Possible new in Vitro Research}

If islands of fresh prostate tissue taken from prostate gland excision for prostate carcinoma and these include only those strictly uncontaminated by $\mathrm{BPH}$ and these are placed in a nutrient solution as used in reference [3] and PLD (commercially available) added, then it is highly likely that the pathology of BPH would be reproduced in these islands. There is however a need to keep calcium levels at a suitable low because higher levels have been shown to prevent epithelial hyperplasia in the past.

There is no intention here of trying to reproduce healthy prostate tissue itself but only to reproduce the pathology of BPH in these islands.

\section{References}

1. Gil-Vernet JM, Alvarez-Vijande R, Gil-Vernet A, Gil-Vernet JM (1944) Ejaculation in men: a dynamic endorectal ultrasonographical study. Br J Urol 73: 442.

2. Christudoss P, Selvakumar R, Fleming JJ, Gopalakrishnan G (2011) Zinc status of patients with benign prostatic hyperplasia and prostate carcinoma. Indian J Urol 27: 14-18. [Crossref]

3. Adolffson Per I.Ahlstrand C. Varenhorst E. Svensson S (2002) Lysophosphatidic acid stimulates proliferation of cultured smooth muscle cells from human BPH tissue: Sildenafil and papaverin generate inhibition. The Prostate 51: 50-58.

4. Sakamoto S, Yokoyama M, Zhang X, Prakash K, Nagao K, et al. (2004) Increased Expression of CYR61, an Extracellular Matrix Signaling Protein in Human Benign Prostatic Hyperplasia and its Regulation by Lysophosphatidic Acid. Endocrinology 145: $2929-2240$

Copyright: (2019 Roper WG. This is an open-access article distributed under the terms of the Creative Commons Attribution License, which permits unrestricted use, distribution, and reproduction in any medium, provided the original author and source are credited. 